\title{
Utilidad de la terapia asistida con perros en la volición de niños con parálisis cerebral en segundo grado de primaria del Colegio la Alegría en el Señor, La Molina, setiembre 2013. Estudio piloto
}

Usefulness of assisted therapy with dogs in volition of children with cerebral palsy, at second grade of the Colegio la Alegría en el Señor, La Molina, september 2013. Pilot study

\author{
Rosario Katherine Flores Cortijo ${ }^{1, a}$, Gina Cecilia Lino Salvador ${ }^{1, a}$
}

\section{RESUMEN}

Objetivo: Evaluar si la terapia asistida con perros es útil en la volición de niños con Parálisis Cerebral en segundo grado de primaria del colegio La Alegría en el Señor. Material y métodos: La presente investigación es de diseño analítico, cuasiexperimental, no aleatorio con la finalidad de valorar la utilidad de la Terapia Asistida con Perros (TAP) en la volición de un grupo de niños con diagnóstico de Parálisis Cerebral (PC). La población final con la que se trabajó estuvo conformada por 6 niños diagnosticados con $\mathrm{PC}$ en $2^{\circ}$ de primaria del colegio La Alegría en el Señor (LAS); se brindó a cada participante 12 sesiones de Terapia Ocupacional utilizando como herramienta terapéutica la TAP. Como instrumento de evaluación se utilizó el Cuestionario Volitivo Pediátrico (PVQ) - Hoja $\mathrm{C}$; este instrumento fue aplicado a cada participante en tres momentos $\left(1^{\circ}, 6^{\circ}\right.$ y $12^{\circ}$ sesión). Resultados: Los resultados obtenidos desde la Sesión I hasta la III muestran incremento progresivo del grado de expresión volitiva, relacionada a la participación del perro de terapia. Finalmente los resultados indican relación entre variables y se muestran a favor de la hipótesis planteada (La TAP es útil en la volición de niños con PC en $2^{\circ}$ grado de primaria del colegio LAS). Los resultados evidencian que los componentes volitivos fueron impactados de forma positiva por la presencia del Perro de Terapia, esto se refleja en los resultados obtenidos, donde finalizado el estudio los indicadores volicionales alcanzaba en el $100 \%$ de niños la etapa de logro. Conclusiones: La TAP es útil para desarrollar la volición de niños con $\mathrm{PC}$ en $2^{\circ}$ de primaria del colegio LAS.

PALABRAS CLAVE: Terapia asistida con animales, volición, parálisis cerebral, terapia ocupacional.

\section{SUMMARY}

Objective: To evaluate if the assisted therapy with dogs is useful in the volition of children with Cerebral Palsy at Second Grade of "La Alegría en el Señor" School. Material an Methods: The current investigation has an analytic, quasi-experimental and no random design in order to assess the usefulness of assisted therapy with dogs (ATD) in volitional development of a group of children diagnosed with cerebral palsy (CP). We worked with a final group of 6 children diagnosed with PC, at Second Grade of "La Alegría en el Señor" School (LASS); each participant was given 12 sessions of occupational therapy using ATD as a therapeutic tool. We used the Pediatric Volitional Questionnaire (PVQ) - Sheet C as evaluation tool; this instrument was administered to each participant in three

Universidad Peruana Cayetano Heredia. Lima, Perú.

a Lic. Tecnólogo Médico - Terapeuta Ocupacional 
different moments (Session 1, 6 and 12). Results: The results from session 1 to 3 show a progressive increase in volitional expression degree, related to the dog's participation in therapy. Finally, the results indicate there is a relationship between variables and that is in favor of the hypothesis (ATD is useful in volitional development of children at Second Grade of LASS). The results prove that components of volition were positively impacted by the dog's presence in therapy; this is reflected by volition indicators which reached the Achievement level in $100 \%$ of children at the end of the study. Conclusions: The ATD is useful for volitional development of children with CP, at Second Grade of "La Alegría en el Señor" School.

KEYWORDS: Animal assisted therapy, volition, cerebral palsy, occupational therapy.

\section{INTRODUCCIÓN}

La parálisis cerebral es una patología con incidencia importante. Según Arteaga et al., la prevalencia global de PC se sitúa aproximadamente entre 2 y 3 por cada 1000 nacidos vivos, los países en desarrollo tienen una prevalencia más elevada, que puede llegar hasta 5 casos por 1000 nacidos; sin que se evidencie una tendencia a disminuir a través de los años (1).

En la actualidad, diversas son las metodologías que se usan para la rehabilitación de pacientes con este diagnóstico, el proceso de rehabilitación suele ser de larga data por lo que con frecuencia se busca la adherencia del paciente y de sus familiares al tratamiento.

Al respecto, el presente estudio investiga un abordaje poco convencional en nuestro medio, que es de la terapia asistida con perros como una herramienta más del terapeuta ocupacional, con el objetivo principal de valorar la utilidad de la terapia asistida con perros en la volición de un grupo de niños con diagnóstico de parálisis cerebral.

La volición puede definirse como un patrón de pensamientos y sentimientos acerca de la propia persona como actor en el propio mundo que ocurren a medida que uno anticipa, elige experimenta e interpreta lo que uno hace (2). La volición en niños con parálisis cerebral, a menudo se ve afectada, esto debido a que, los niños que crecen con una discapacidad aprenden que no pueden hacer lo que los otros hacen y son propensos a desarrollar sentimientos de ineficacia de modo que, estos niños pueden volverse innecesariamente dependientes de otros, porque no ven sus propias acciones como la vía más eficaz para lograr sus deseos (3) ,y desarrollar así su sentido de capacidad y autoeficacia.

La terapia asistida con perros es una técnica, herramienta o complemento a una terapia concreta, según el manual de terapia asistida con animales , podemos denominar una intervención terapéutica como Terapia Asistida con Animales (TAA), si ésta cuenta con "un triángulo de trabajo formado por el profesional de la salud, el instructor del animal yel animal" (4). Deben contar con objetivos concretos y criterios de evaluación. Los perros son comúnmente los más utilizados en la terapia asistida con animales; son excelentes ayudantes de terapia, aman a las personas independientemente de su aspecto, problemas o condición social (5). Además es un "facilitador social, aumenta la motivación del paciente haciendo de facilitador emocional, parecen disfrutar trabajando y fácilmente aprenden tareas muy específicas, se adaptan bien para nuevas tareas, tienden a ser muy atentos a sus compañeros humanos primarios, es algo natural para ellos servir como entes sociales, conocer y saludar a gente nueva. Esta característica puede ser una buena base para un programa de TAA (6).

La investigación que a continuación se presenta plantea como hipótesis que la terapia asistida con perros es útil en la volición de niños con parálisis cerebral en segundo grado de primaria del colegio La Alegría en el Señor. Fue ejecutada en el mes de Setiembre del año 2013 en el distrito de la Molina, Lima. La población final con la que se trabajó estuvo conformada por seis niños diagnosticados con Parálisis Cerebral en segundo grado de primaria del colegio La Alegría en el Señor.

En virtud de los resultados obtenidos creemos que la inclusión de un can como instrumento de terapia, permitiría mayor participación voluntaria del niño en su tratamiento, por ser en general, este animal, un importante elemento motivacional en la participación ocupacional; así la Terapia Asistida con Perros puede ser una nueva alternativa Terapéutica en nuestro país (tal como se está dando en países vecinos) $\mathrm{y}$ así enriquecer la labor que realiza el Terapeuta Ocupacional. 


\section{MATERIAL Y METODOS}

El diseño de estudio de la presente investigación es analítico, cuasi experimental, con un diseño muestral no aleatorio.

La población de estudio está dada por los niños diagnosticados con parálisis cerebral, que cursan segundo grado de primaria del colegio La Alegría en el Señor; considerando los criterios de inclusión y exclusión el número final de participantes fue igual a 6 niños (de cuyos padres/apoderados se contó con el consentimiento informado).

Nuestra intervención fue realizada en doce sesiones, con una frecuencia de tres veces por semana, cada una de ellas fue diseñada: Según los objetivos de tratamiento de los menores en terapia ocupacional; es importante resaltar que los objetivos de intervención fueron determinados por el terapeuta de planta siguiendo su tratamiento común con objetivos pre establecidos para cada usuario, estos objetivos son independientes a los que se plantea en el actual estudio, pues únicamente se introdujo al Perro de Terapia con el propósito de valorar la utilidad de la Terapia Asistida con Perros en la volición de niños con Parálisis Cerebral en su rutina terapéutica. Y según el marco de trabajo de Terapia Asistida con Animales siguiendo los Parámetros del Manual de Terapia Asistida con Animales Fundación Bocalán España - Asociación Bocalán Perú.
Se utilizó como instrumento de evaluación la Hoja C del Cuestionario Volitivo Pediátrico (Pediatric Volitional Questionnaire [PVQ]); el que fue aplicado a cada participante en tres momentos $\left(1^{\circ}, 6^{\circ}\right.$ y $12^{\circ}$ sesión $)$ para finalmente, contrastar resultados y registrar evidencias objetivas, además la hoja de Registro de las Características Ambientales de dicho cuestionario sirvió para identificar las condiciones ambientales (dentro de éstas se considera la participación del perro de terapia) en las que se dieron los diferentes cambios de los indicadores volitivos.

Se elaboró una base de datos en el programa Microsoft Excel; el cual se utilizó para ingresar los resultados obtenidos. Posteriormente dichos datos fueron analizados a través del uso de medidas de tendencia central (comparación y verificación de los resultados) con lo cual se realizaron los cuadros y gráficascorrespondientes. Para analizar el objetivo que mide la relación entre variables fue necesario agrupar los datos en una tabla de contingencia en Microsoft Excel, los mismos que fueron exportados a SPSS IBM STADISTIC 19, con el objetivo de calcular los valores Chi cuadrado y determinar el nivel de relación entre variables y así establecer los valores de significancia válidos para la investigación y además determinar la influencia de la presencia del perro con respecto a la volición de los participantes.

Tabla 1. Comparativa de resultados de las tres sesiones de evaluación

\begin{tabular}{|c|c|c|c|c|c|c|c|c|c|c|c|c|c|c|c|}
\hline \multirow{3}{*}{ Indicador Volicional } & \multicolumn{5}{|c|}{ Evaluacion 1} & \multicolumn{5}{|c|}{ Evaluacion $\|$} & \multicolumn{5}{|c|}{ Evaluacion III } \\
\hline & \multicolumn{5}{|c|}{ Grado de expresión de la volición } & \multicolumn{5}{|c|}{ Grado de expresión de la volición } & \multicolumn{5}{|c|}{ Grado de expresión de la volición } \\
\hline & E\% & \% & $D \%$ & P\% & NA\% & E\% & $\%$ & D\% & $P \%$ & NA\% & E\% & $\mathbf{H}$ & $D \%$ & $\mathrm{P} \%$ & NA\% \\
\hline (1) Mestra Curiosidad & 100 & & & & & 100 & & & & & 100 & & & & \\
\hline 2) ricia Acciones & 50 & 33 & & 17 & & 83 & 17 & & & & 100 & & & & \\
\hline 3) Se Orienta hacia un Obietivo & 50 & 33 & 17 & & & 100 & & & & & 100 & & & & \\
\hline 4) Musta Préterencias & 33 & 50 & & & 17 & 83 & 17 & & & & 100 & & & & \\
\hline 5) Itrenta Cosas Nueras & 33 & 67 & & & & 67 & 33 & & & & 100 & & & & \\
\hline i) Permaneces hroulucrado & 83 & 17 & & & & 100 & & & & & 100 & & & & \\
\hline 7) Expresa Pacer con sus Logros & 83 & 17 & & & & 100 & & & & & 100 & & & & \\
\hline 8) Trata de Rescover Problemas & 50 & 17 & & & 33 & 100 & & & & & 100 & & & & \\
\hline 9) Triala de Produci Electos & 17 & 33 & & 17 & 33 & 50 & 17 & & & 33 & 83 & 17 & & & \\
\hline 10) Pracicica Hebilidades & 33 & 50 & & & 17 & 100 & & & & & 100 & & & & \\
\hline (1) Busca Desafios & 17 & 17 & & & 67 & 50 & 17 & & & 33 & 100 & & & & \\
\hline 12) Organiza/ / Nodficica e Ambiente & & & & & 100 & 17 & & 17 & & 67 & 66 & 17 & & & 17 \\
\hline 13) Realiza una Acivhasia Compl. & 83 & 17 & & & & 100 & & & & & 100 & & & & \\
\hline (1) Usa la maginación & & 50 & 17 & & 33 & 33 & 67 & & & & 100 & & & & \\
\hline
\end{tabular}




\section{RESULTADOS}

La tabla 1 resume en porcentajes los grados de expresión volitiva que la población de estudio fue manifestando a lo largo del estudio, así se evidencia un grado progresivo de la expresión volitiva en los distintos indicadores volicionales. De esta forma podemos observar como a lo largo de las sesiones el porcentaje de población que evidencia grados de expresión volitiva más bajos (pasivo, dudoso, involucrado) decrece y así el porcentaje de población que muestra grado de expresión volitiva más alto (espontáneo) incrementa.

La tabla 2 muestra específicamente los valores de los grados de expresión volitiva obtenidos en la evaluación final. Por tanto siguiendo lo establecido por la Hoja C del cuestionario volitivo pediátrico, la presenta tabla evidencia que finalizada la investigación, todos los niños incrementaron su sentido de capacidad y control; pues los grados de expresión volitiva de la población de estudio se muestran incrementados tanto en los indicadores correspondientes a la etapa de Exploración, como en la de Competencia, de este modo finalmente el desarrollo volitivo de los participantes supera las dos etapas descritas y alcanza una etapa superior de desarrollo volitivo que viene a ser la etapa de Logro.

El gráfico 1 muestra que la creciente interacción con el perro de terapia propició el grado progresivo de expresión volitiva de los niños en sus sesiones terapéuticas, es así que tal como se observa en la gráfica: en la sesión I un 17\% de la población expresa su volición en grado dudoso, y un $83 \%$ en grado involucrado y $0 \%$ en grado espontáneo; en la sesión II el $0 \%$ de la población expresa su volición en grado dudoso, $67 \%$ en grado involucrado y un $33 \%$ en grado espontáneo y finalmente en la sesión III el 0\% de la población expresa su volición en grado dudoso,

Tabla 2. Evaluación Final (Evaluación III).

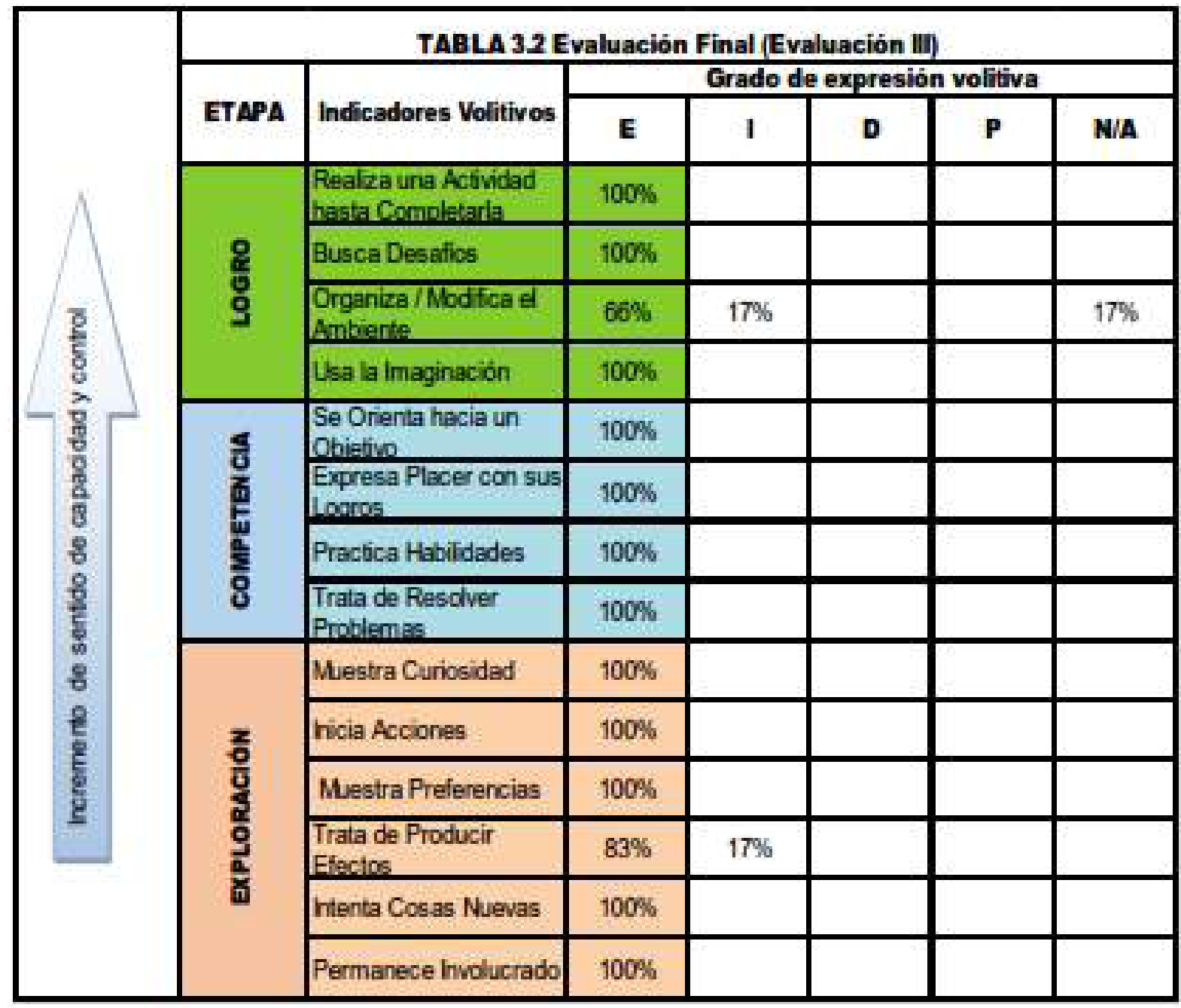




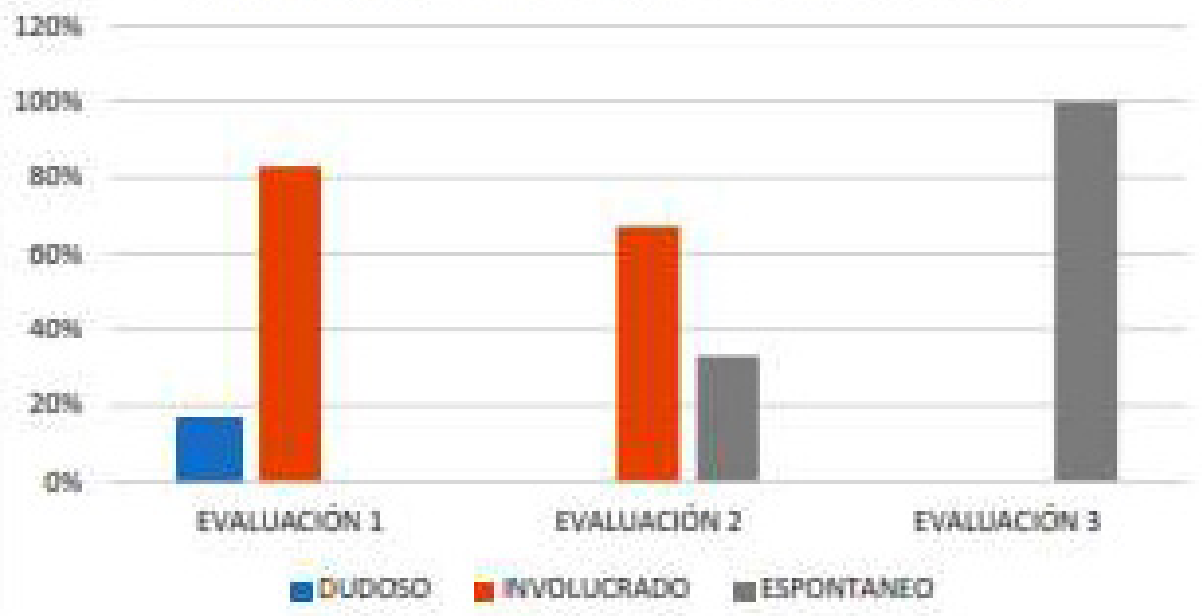

Gráfico 1. Variaciones de la volición

el $0 \%$ en grado involucrado, y el $100 \%$ en grado espontáneo. Los resultados obtenidos indican relación entre variables y se muestran a favor de la hipótesis planteada: "La terapia asistida es útil en la volición de los niños con parálisis cerebral del colegio la Alegría en el Señor".

El impacto positivo en la volición de los niños participantes relacionado a la participación del can dentro de las sesiones terapéuticas (es decir terapia asistida con perro), se justifica en la relación chi $^{2}$ aplicado al estudio: Donde, trabajando con un nivel de confianza al 95\% (con $\alpha=0,05)$; se observa que el chi ${ }^{2}$-calculado $(13,67)$,resulta mayor que el chi ${ }^{2}$-tabla $(9,49)$; debido a que el Chi Calculado se encuentra dentro de la región de rechazo por lo que se rechaza la hipótesis nula $(\mathrm{Ho}=\mathrm{La}$ terapia asistida no es útil en la volición de los niños con Parálisis Cerebral del colegio la Alegría en el Señor) y se acepta la hipótesis alternativa ( $\mathrm{H} 1=\mathrm{La}$ terapia asistida es útil en la volición de los niños con parálisis cerebral del colegio la Alegría en el Señor), es decir se acepta la hipótesis planteada en la presente investigación; demostrando así en el estudio la relación entre variables.

\section{DISCUSION}

O`Sullivan indica que los efectos producidos en el sistema autónomo parasimpático pueden influir en la adaptación del tono (7).

Nuestro estudio nos ayuda a corroborar que la interacción con un perro de terapia efectivamente produjo algún efecto favorable a este nivel, ya que evidenciamos un efecto calmante, disminución de las respuestas de lucha; que en suma aportaron en la adaptación de tono lo cual contribuyó a que los niños se sientan más motivados por el hacer.

Nimer y Lundahl, concluyen que la TAA se muestra prometedora al adicionarse a las intervenciones tradicionales (7), afirmación a la que nos aunamos tras los resultados obtenidos.

Este trabajo evidencia significativo impacto favorable en la volición de nuestra población de estudio, lo que brinda un punto de partida relevante para futuras investigaciones en el campo de la Terapia Ocupacional asistida con animales.

\section{CONCLUSIONES}

La Causalidad personal, Valores e Intereses de los niños fueron impactados de forma positiva por la presencia del Perro de Terapia debido a que hubo una influencia directa en los pensamientos y sentimientos volitivos, lo que explica la creciente motivación por el hacer, esto se refleja en los resultados obtenidos, donde finalizado el estudio los diferentes indicadores volicionales en promedio alcanzaba en el $100 \%$ de niños la etapa de logro.

Por tal motivo concluimos que la terapia asistida con perros es útil para desarrollar la volición de niños con Parálisis Cerebral en segundo grado de primaria del colegio La Alegría en el Señor.

Por otra parte, las emociones generadas por el contacto directo o indirecto con el animal, originaron comportamientos que los comprometieron a actuar con un significado lógico y además promovieron el incremento del sentido de capacidad personal y 
control en toda la población de estudio, lo cual se evidenció en la participación más activa de los niños en la intervención terapéutica y en la disposición para el uso de sus habilidades y el disfrute de hacerlo, para enfrentar satisfactoriamente las demandas de las actividades asistidas con el Perro de Terapia.

El estudio evidenció que los indicadores volicionales en su totalidad atravesaron variaciones favorables, éstas mostraron que los tres niveles de cambio volicional: "exploración, competencia y logro", fueron influenciados de manera positiva; debido a que la terapia asistida con perros otorga al niño el constante deseo de interactuar con el ambiente (favoreciendo de este modo la etapa de exploración en los niños); así también el abordaje mencionado, facilita la incorporación de nuevas maneras de hacer las cosas aprendidas en la etapa anterior, es decir facilita la "competencia", pues los niños buscaron interactuar activamente con el ambiente y a la vez se esforzaron por ejercer influencia sobre éste (dictaban comandos al animal hasta que éste logre hacer lo que los niños esperaban, invertían esfuerzo para realizar de manera exitosa una actividad o tarea dedicada al animal y disfrutaban del éxito obtenido); mostrando un desempeño consistente y satisfactorio lo que permitió alcanzar la etapa de "logro".

La presencia del perro de terapia y la creciente interacción con éste propició el grado progresivo de expresión volitiva de los niños en sus sesiones terapéuticas. En la intervención ejecutada, el perro se convirtió en una herramienta singular, que no solamente cumplió el papel de un objeto interesante cualquiera, sino que la simple presencia del perro suscitó en los niños a interactuar con éste; y conforme se fueron incrementando las oportunidades de interacción con el animal (mayor número de sesiones) los vínculos generados propiciaron en los usuarios mayor interés por participar activamente en las sesiones terapéuticas. Las condiciones ambientales dadas durante el proceso de la intervención, si bien fueron similares durante todo el proceso, tras cada etapa de evaluación, el uso de la hoja de registro de características ambientales permitió identificar y cambiar situaciones o condiciones que restringían o limitaban de algún modo la expresión volitiva de los participantes (por ejemplo mejorar la ubicación de la evaluadora y voluntarios durante las sesiones de terapia para no distraer o intimidar a los participantes susceptibles a ello, limitar el número de objetos que manejaban si esto los desorganizaban, a su vez ubicar a los materiales en superficies más accesibles a los niños y menos accesibles al animal para mejorar el orden de la sesión y evitar distracción del perro de terapia -por ejemplo con la comida-, etc.) así finalmente las condiciones ambientales en las que se desarrolló la presente investigación, en sus cuatro secciones (espacio, objetos, ambiente social y formas ocupacionales) giraron en relación a la figura y constancia del animal, además estuvieron directamente relacionadas con el trabajo que se ejercía con el perro de terapia (por ejemplo: objetos y formas ocupacionales diseñados para enseñar, cuidar, alimentar, vestir, jugar, celebrar, etc. con el perro de terapia); esta diversidad de condiciones, si por lo menos no fue constante, dirigieron favorablemente la acción de cada niño, pues brindaron variedad de oportunidades que nutrían la expectativa de los participantes por lo que se evidenciaron conductas particulares (como esforzarse para realizar ajustes posturales más precisos y frecuentes, mayor uso de la comunicación oral dirigida e intencionada, interrelación social más fluida, entre otros) que los propios niños buscaban sostener para mantener una participación activa y desempeño favorable en su terapia.

\section{Correspondencia}

Rosario Katherine Flores Cortijo

Correo electrónico: kathyros_2307@hotmail.com

\section{REFERENCIAS BIBLIOGRÁFÍCAS}

1 Taboada L, Quintero K, Casamajor M.,et al. (2013). Epidemiología de la parálisis cerebral en el Estado Plurinacional de Bolivia, 2009-2012. Revista Peruana de Epidemiología. 2013; 17(2):0-0.

2. Kielhofner G. Terapia ocupacional modelo de ocupación humana teoría y aplicación. Buenos Aires, Argentina: Editorial Médica Panamericana; 2004.

3. Bouyt B, Gillen G, Scaffa M. Willard \& Spackman Terapia Ocupacional. Buenos Aires, Argentina: Médica Panamericana; 2005.

4. Fundación Bocalán España. Manual de Terapia Asistida con Animales: II Curso de formación en Terapia Asistida con Animales Lima-Perú 20122013. Lima, Perú: Asociación Bocalán Perú 2012.

5. Burch M. Volunteering with your pet, how to get involved in animal-assisted therapy with any kind of pet. New York: Howell books; 1996.

6. Aubrey F. Animal assisted therapy theoretical Foundations and Guidelines for Practice. California: Academic Press; 2000.

7. Moscoso F. (2009). Caninoterapia para la atención de pacientes con evento cerebrovascular - estudio piloto. Tesis de maestría. Bogota: Universidad Sergio Arboleda; 2009. 